\title{
Study on the Elman Neural Network Operation Control Strategy of the Central Air Conditioning Chilled Water System*
}

\author{
Jianwei Li1,2, Qingchang Ren³, Hai Long ${ }^{4}$, Zengxi Feng3 \\ ${ }^{1}$ School of Civil Engineering, Xi'an University of Architecture \& Technology, Xi'an, China \\ ${ }^{2}$ China Nuclear Power Technology Research Institute, Shenzhen, China \\ ${ }^{3}$ School of Information \& Control Engineering, Xi'an University of Architecture \& Technology, Xi'an, China \\ ${ }^{4}$ China Nuclear Power Operations Co., Ltd., Shenzhen, China \\ Email: lijianwei329@163.com
}

How to cite this paper: Li, J.W., Ren, Q.C., Long, H. and Feng, Z.X. (2019) Study on the Elman Neural Network Operation Control Strategy of the Central Air Conditioning Chilled Water System. World Journal of Engineering and Technology, 7, 73-82.

https://doi.org/10.4236/wjet.2019.72B009

Received: February 21, 2019

Accepted: May 26, 2019

Published: May 29, 2019

\begin{abstract}
The stable operation of the central air conditioning water system always is a major difficulty for the control profession. Paper focus on the water system with multi variable, strong coupling, nonlinear, large time delay characteristics, presented use feed forward coupling compensation method, to eliminate the coupling effect between temperature and pressure. In this paper, the Elman neural network controller is designed for the first time, and the simulation results show that the response time of Elman neural network controller is shorter, the system is more stable and the overshoot is small.
\end{abstract}

\section{Keywords}

Feed Forward Coupling Compensation, Central Air Conditioning Water System Always, Temperature Difference Control, Pressure Difference Control, Elman Neural Network

\section{Introduction}

For the operation control of the central air conditioning water system, generally control the temperature difference and pressure difference. More scholars have done a lot of experimental research and engineering verification on the control method for temperature difference and pressure difference [1]. K. F. Fong optimization temperature setpoint of chilled water by EP genetic algorithm, water pump control strategy of air conditioning water system are analyzed and experimental by Brian J. Moore and Jamess B. [2] [3]. For the control strategy and ${ }^{\star}$ The natural science fund of Shanxi province education office (15JK1389). 
control algorithm of the control circuit 2 input -2 output water system, the research is very few, so this paper design a Elman neural network controller for controlling the air conditioning water system stable operation by feed forward coupling compensation [4] [5] [6].

\section{Chilled Water System Model}

As Figure 1 show, for the operation control of the central air-conditioning water system, mainly through the control of chilled water supply and return water loop pressure and temperature to achieve. Chilled water supply and return water loop pressure is mainly through the bypass valve to achieve, the temperature difference is mainly through the control of chilled water flow to achieve. The specific control system diagram is shown in Figure 2 and Figure 3 [7].

As Fiure 2 show, for a given chilled water supply and return water loop pressure difference value, mainly through the opening of the regulating valve to achieve the loop of the chilled water supply and return water pressure control, when the chilled water supply and return water loop differential pressure differential pressure is lower than the setpoint, the bypass valve opening decreases. In order to increase the impedance of the pipeline, so as to achieve the stable pressure, and finally achieve the system running requirements.

As Figure 3 show, for a given temperature of chilled water supply and return water loop setpoints, mainly through adjusting the pump speed to change the operation flow of the chilled water system, to get the supply and return water temperature of chilled water loop control, when the temperature difference between chilled water supply and return water temperature is lower than the setpoint of the loop, by reducing the speed water pump. To reduce operation flow chilled water system, to achieve the temperature stability.

In fact, because of the strong direct coupling between the differential pressure regulating and the temperature regulating system, so it is very difficult to control

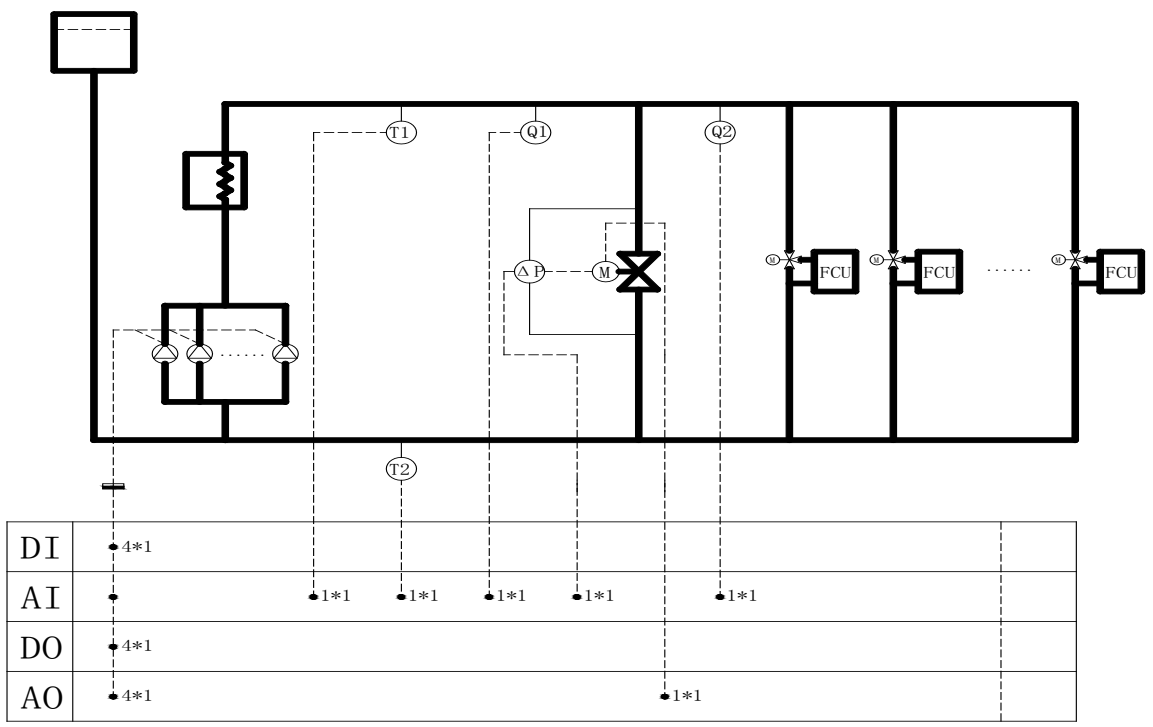

Figure 1. Control signal point of central air conditioning chilled water system. 


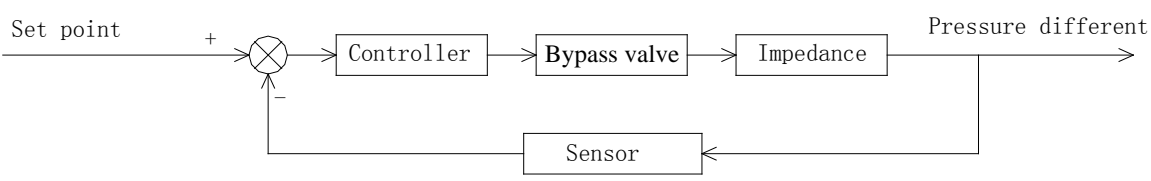

Figure 2. Control system of chilled water pressure difference.



Figure 3. Temperature difference control system of chilled water.

the system. As shown in Figure 4, when the different pressure regulating opening while changing the bypass valve, also changed the impedance of chilled water piping system, resulting in the chilled water system flow changes in the system under a certain load, the inevitable change of chilled water supply and return water temperature loop. Similarly, when the temperature difference is adjusted by changing the pump the rotational speed of the flow change at the same time, also cause the pressure difference on both sides of the chilled water bypass valve, and the impact of the water system pressure control.

In fact, to central air-conditioning chilled water control system, is a "2 input -2 output" control system. As show in Figure $5, G_{11}(s)$ as the bypass valve opening-pipeline pressure control loop model, $G_{21}(s)$ as the bypass valve opening-supply and return temperature control loop model, $G_{12}(s)$ as the pump speed-line pressure control loop model, $G_{22}(s)$ for the "water pump speed for the return water temperature control loop model, $\mathrm{y}_{1}$ for chilled water loop pipeline pressure, $\mathrm{y}_{2}$ for chilled water supply and return temperature for the $r_{1}$ loop, chilled water loop pressure difference value, $r_{1}$ for chilled water supply and return temperature setting circuit. With the method of system identification (using MATLAB system identification toolbox), can get the mathematical model of each circuit module, as follows:

$$
\begin{gathered}
G(s)=\left[\begin{array}{ll}
G_{11}(s) & G_{12}(s) \\
G_{21}(s) & G_{22}(s)
\end{array}\right]=\left[\begin{array}{ll}
\frac{Y_{1}(s)}{U_{1}(s)} & \frac{Y_{1}(s)}{U_{2}(s)} \\
\frac{Y_{2}(s)}{U_{1}(s)} & \frac{Y_{2}(s)}{U_{2}(s)}
\end{array}\right] \\
\frac{Y_{1}(s)}{U_{1}(s)}=\frac{0.0000274 s+0.0002629}{s^{2}-2.0043 s+1.0054} e^{-7.9 s} \\
\frac{Y_{2}(s)}{U_{1}(s)}=\frac{0.0000025 s-0.0000022}{s^{2}-2.0043 s+1.0054} e^{-12.2 s} \\
\frac{Y_{1}(s)}{U_{2}(s)}=\frac{0.1105 s+0.5013}{s^{2}-2.0043 s+1.0054} e^{-20.1 s} \\
\frac{Y_{2}(s)}{U_{2}(s)}=\frac{0.0053 s-0.0047}{s^{2}-2.0043 s+1.0054} e^{-16.8 s}
\end{gathered}
$$






Figure 4. Coupling relationship of chilled water pressure difference and temperature difference.



Figure 5. Coupling relationship of air conditioning water system control.

\section{Decoupling of Water System of Central Air Conditioning System and Design of Controller}

\subsection{Control Model Decoupling of Chilled Water System}

For the decoupling method of the chilled water control model, the traditional decoupling method mainly has the modern frequency method and the feed forward compensation method. The modern frequency method also includes time domain method. The pre compensation method includes the invariance of the contact, the matrix inversion and the inverse decoupling. In this paper, the decoupling network is designed by using the invariance principle of feed forward decoupling compensation method, as shown in Figure 6:

Order:

$$
\begin{aligned}
& \mathrm{y}_{11}-\mathrm{y}_{12}=0\left(\mathrm{~m}_{2} \neq 0\right) \\
& \mathrm{y}_{21}-\mathrm{y}_{22}=0\left(\mathrm{~m}_{1} \neq 0\right)
\end{aligned}
$$

So:

$$
\begin{aligned}
& G_{12}(s)-D_{12}(s) G_{11}(s)=0 \\
& G_{21}(s)-D_{21}(s) G_{22}(s)=0
\end{aligned}
$$

So the mathematical model of the decoupling network: 


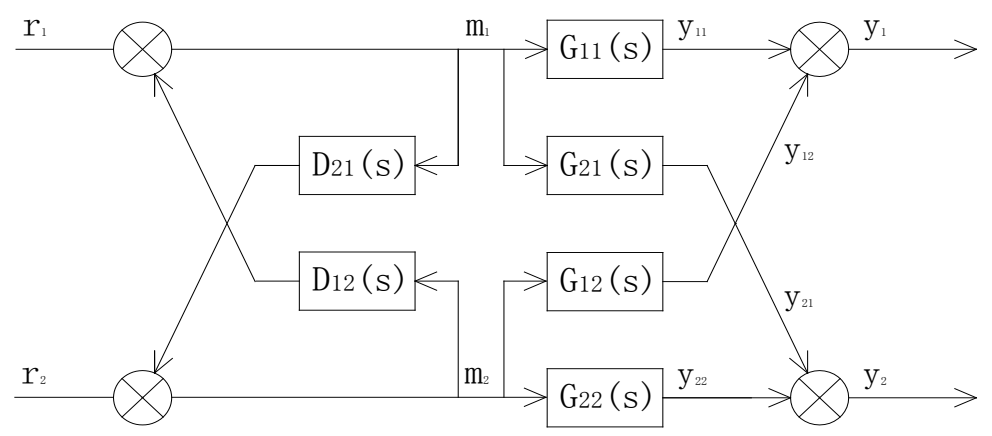

Figure 6. feedforward compensation decoupling network.

$$
\begin{aligned}
& D_{12}(s)=-\frac{G_{12}(s)}{G_{11}(s)} \\
& D_{21}(s)=-\frac{G_{21}(s)}{G_{22}(s)}
\end{aligned}
$$

Put expressions (1) input (2), (3):

$$
\begin{gathered}
D_{12}(s)=\frac{9.12 s-8.03}{s+9.59} e^{-4.3 s} \\
D_{21}(s)=\frac{20.85 s-94.59}{s-0.89} e^{-3.3 s}
\end{gathered}
$$

\subsection{Elman Neural Network Controller}

Figure 6 build the " 2 input -2 output" decoupling network, although solved the problem of coupling between systems, but in order to ensure that the system output value satisfies the set value requirements, also need to set up the network controller based on feed forward compensation decoupling in Figure 6. In the control system, the PID controller is the most commonly used controller. Its principle block diagram is shown in Figure 7.

PID controller is based on the control deviation which on value $\operatorname{Rin}(t)$ and the Yout(t) :

$$
\begin{gathered}
\operatorname{Error}(\mathrm{t})=\operatorname{Rin}(\mathrm{t})-\operatorname{Yout}(\mathrm{t}) \\
u(t)=k_{p}\left(\operatorname{error}(t)+\frac{1}{k_{i}} \int_{0}^{t} \operatorname{error}(t) d t+\frac{k_{d} \operatorname{derror}(t)}{d t}\right) \\
G(s)=\frac{U(s)}{E(s)}=k_{p}\left(1+\frac{1}{k_{i} s}+k_{d} s\right)
\end{gathered}
$$

where:

$K_{p}$ : ratio coefficient;

$K_{i}$ integral time constant;

$K_{d}$ differential time constant.

In the actual project cases, $k_{p} \quad k_{i} k_{d}$ control parameter values have great influence on the output of the system, because of the central air conditioning system of "multi input and multi output, strong coupling between the system 




Figure 7. Diagram of PID controller.

and other factors, resulting in the actual debugging of central air-conditioning system, control tuning parameters, $k_{p} k_{i} k_{d}$ has become very difficult. Therefore, the control loop of the water system of the central air conditioning system, the choice of the corresponding PID parameters becomes very important. If the parameter selection is not properly, it will not only lead to oscillation of the control loop, and even cause the instability of the control loop of the whole air conditioning system. The selection of control parameters $k_{p} k_{i} k_{d}$ in the actual air conditioning control case is also the difficulty of the project. In this paper, a new method of PID parameter $k_{p} \quad k_{i} k_{d}$ is proposed, which uses the Elman neural network to adjust the parameters of the PID controller $k_{p} k_{i}$ $k_{d}$. As shown in Figure 8.

\subsection{Elman Neural Network Architecture}

Figure 9 for the Elman neural network model structure, from Figure 9, we can see that the model design of the algorithm for the 3 input, 3 output, the middle layer for the 5 layer structure.

\subsection{Model Training Steps}

1) Initialization model: $w_{j l} \quad w_{i j} \quad w_{k j} \quad \theta_{l} \quad \theta_{j}$;

2) Input training values: $x^{1}, x^{2} \cdots x^{p}$ the output value of the model: $y^{1}, y^{2} \cdots y^{p}$

3) estimate: $\left|t^{p}-y^{p}\right|<\varepsilon$;

4) If the step (3) is satisfied, the weight and the threshold value of the model are corresponding to the model, or the following steps:

5) The following values are calculated

$$
\begin{gathered}
E_{p l}=\frac{1}{2} \sum_{l=0}^{m-l}\left(t_{l}^{p_{l}}-y_{l}^{p_{l}}\right)^{2} ; \\
E_{a l l}=\frac{1}{2} \sum_{p_{l}=1}^{p} \sum_{l=0}^{m-l}\left(t_{l}^{p_{l}}-y_{l}^{p_{l}}\right)^{2} ; \\
w_{j l}(n+1)=w_{j l}(n)-\eta \frac{\partial E_{a l l}}{\partial w_{j l}} ; \\
\theta_{l}(n+1)=\theta_{l}(n)-\eta \frac{\partial E_{a l l}}{\partial \theta_{l}} ; \\
w_{i j}(n+1)=w_{i j}(n)-\eta \frac{\partial E_{a l l}}{\partial w_{i j}} ;
\end{gathered}
$$




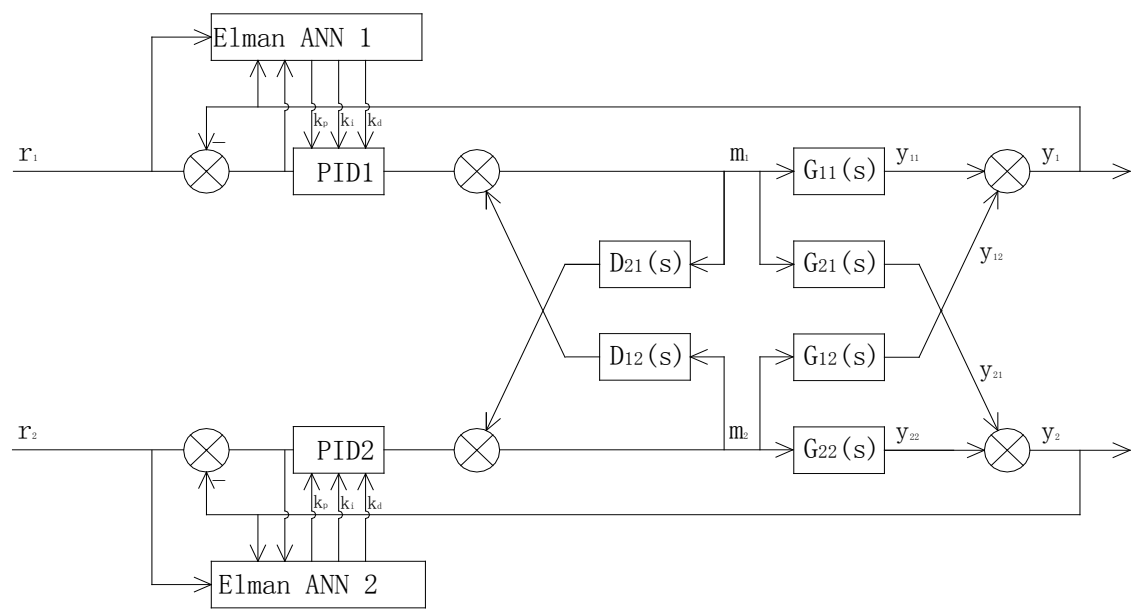

Figure 8. The structure of PID control based on Elman neural network.



Figure 9. Structure of Elman neural network model.

$$
\begin{gathered}
w_{k j}(n+1)=w_{k j}(n)-\eta \frac{\partial E_{a l l}}{\partial w_{k j}} ; \\
\theta_{j}(n+1)=\theta_{j}(n)-\eta \frac{\partial E_{a l l}}{\partial \theta_{j}}
\end{gathered}
$$

6) calculated the model again: $\begin{array}{llllll}w_{j l} & w_{i j} & w_{k j} & \theta_{l} & \theta_{j}\end{array}$

7) Return step (2)

If the step (3) satisfies the condition and stops the training (step 5), the measured value is input to the model, and the predicted value is obtained.

\section{Simulation and Analysis of Water System Operation Control}

\subsection{Elman Neural Network Controller Program Simulation}

According to the analysis of simulation program controller of central air conditioning water system, this paper uses MATLAB software as a simulation tool for research, program can be realized by MATLAB in Simulink, can be directly through the MATLAB program simulation directly.

As shown in Figure 10, for the Elman neural network PID control algorithm simulation diagram, procedures in accordance with the Elman neural network 




Figure 10. Elman neural network PID control algorithm simulation.

PID control structure based on given the formula (1), (12), (13) the corresponding coefficients corresponding simulation chart module. And the preparation of Elman PID1 and Elman PID2 corresponding to the Elman neural network PIDcontrol operation of the $\mathrm{m}$ file.

\subsection{Simulation Results of Elman 4.2. Neural Network Controller}

Figure 11 shows the Elman neural network PID pressure control corresponding to the input-output simulation results, it can be seen from Figure 11, the Elman neural network PID control based on differential pressure, can be very good to achieve the central air conditioning water system pressure difference value of precision control, but the overshoot of Elman neural network PID differential pressure control based on the comparison, the output of the system in a short time quickly from the pressure difference of $0 \mathrm{~m}$ water column, up to $17.137 \mathrm{~m}$ water column, so the central air conditioning water system is bad, need to give enough attention in the subsequent engineering verification, but also need to see that the Elman neural network PID differential controllers based on the output of the system rise time is very short, the rise time is only $7 \mathrm{~S}$, the adjusting time is only $16 \mathrm{~S}$, and can be quickly to ensure the system output stable.

Figure 12 shows the Elman PID neural network temperature control corresponding to the input-output simulation results, it can be seen from Figure 12, the PID temperature control based on Elman neural network, the output of the system can not only quickly change follow the preset temperature value changes, and the system is stable, small overshoot. Thus, based on the Elman neural network PID temperature control, can achieve very good operating results.

In practical projects, for the regulation of central air conditioning water system operation, but also to adjust the temperature and pressure regulation by using the temperature difference, therefore, Elman neural network PID differential 


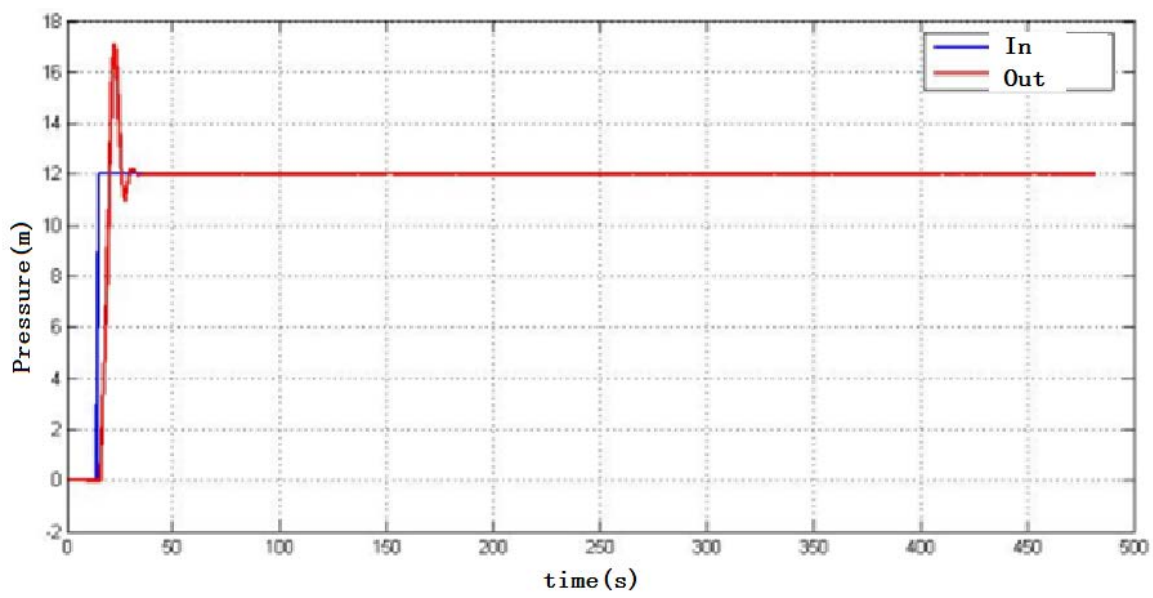

Figure 11. Elman neural network PID pressure control corresponding to the input-output simulation results.



Figure 12. Elman neural network PID temperature difference control corresponding input output simulation results.

pressure control, can realize the central air conditioning water system operation good regulation.

\section{Summary}

In view of the central air conditioning water system control " 2 input -2 output" characteristics, using the invariance principle of feed forward decoupling compensation method, realize the decoupling network structure of the central air conditioning water system "2 input -2 output", and the use of Elman neural network based on PID control algorithm, to achieve the precise control of the central air conditioning water system pressure difference the temperature difference, the simulation results show that Elman neural network control algorithm based on PID, not only can quickly respond to changes in input system, and the control precision is high, operation results are stable and have good application value. 


\section{Conflicts of Interest}

The authors declare no conflicts of interest regarding the publication of this paper.

\section{References}

[1] Fonf, K.F., Hanby, V.I. and Chow, T.T. (2010) HVAC System Optimization for Energy Management by Evolutionary Programming. Energy and Building, 38, 220-231. https://doi.org/10.1016/j.enbuild.2005.05.008

[2] Moore, B.J. and Scott Fisher, D. (2008) Pump Differential Pressure Setpoint Reset Based on Chilled Water Valve Position. ASHRAE Transactions, 373-379.

[3] Rishei, J.B. (2008) Control of Variable Speed Pumps for HVAC Water Systems. ASHRAE Transactions, 380-389.

[4] Li, J.W. and Feng, Z.X. (2014) Energy Conservation Optimization and Tracking Algorithm Based on Adaptive Fusion GA Model. Journal of Computational Information Systems, 10, 8857-8864.

[5] Beheshti, Z. and Shamsuddin, S.M.H. (2014) Centripetal Accelerated Particle Swarm Optimization. Information Sciences, 258, 54-79. https://doi.org/10.1016/j.ins.2013.08.015

[6] Li, J.W. and Feng, Z.X. (2016) Application of the Circuit Theory to the High-Precision Pressure Regulation System in Nuclear Power Plants. Journal of Computational and Theoretical Nanoscience, 3, 1852-1860.

https://doi.org/10.1166/jctn.2016.5123

[7] Zhao, J., et al. (2014) Energy plus Model-Based Predictive Control within Design-Build-Operate Energy Information Modelling Infrastructure. Journal of Building Performance Simulation, 8, 1-14. ttps://doi.org/10.1080/19401493.2014.891656 